Tianyu Li, Xindi Xu, Meifen Huang, Man Wang and Qiong Wu*

\title{
Crystal structure of tris(azido- $\left.\mathrm{K}^{1} N\right)-(\mathrm{N}$ - (2-aminoethyl)- $\mathrm{N}$-methyl-1,3-propanediamine- $\left.\mathrm{K}^{3} N, N^{\prime}, N^{\prime \prime}\right)$ cobalt(III), $\mathrm{C}_{7} \mathrm{H}_{19} \mathrm{CoN}_{12}$
}

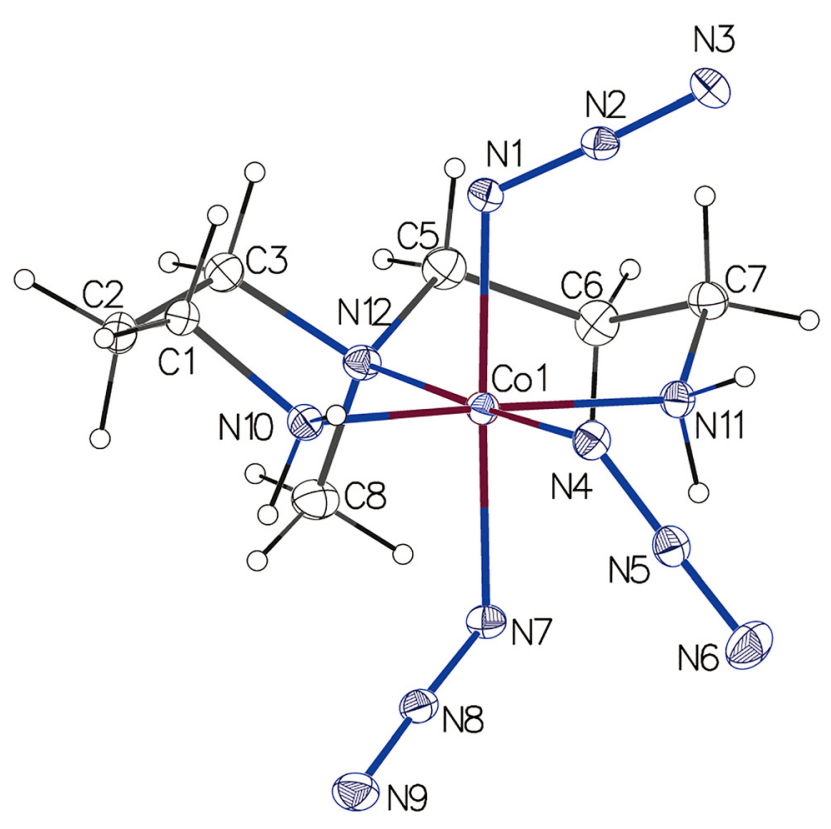

https://doi.org/10.1515/ncrs-2020-0544

Received October 21, 2020; accepted November 29, 2020; published online December 10, 2020

\begin{abstract}
$\mathrm{C}_{7} \mathrm{H}_{19} \mathrm{CoN}_{12}$, monoclinic, $P 2_{1} / c$ (no. 14), $a=7.7111(2) \AA$, $b=10.9909(3) \AA, c=17.5650(4) \AA, \beta=113.7570(10)^{\circ}$, $V=1362.52(6) \AA^{3}, Z=4, R_{g t}(F)=0.0266, w R_{r e f}\left(F^{2}\right)=0.0718$, $T=150(2) \mathrm{K}$.
\end{abstract}

CCDC No.: 2039151

The molecular structure is shown in the Figure. Table 1 contains crystallographic data and Table 2 contains the list of the atoms including atomic coordinates and displacement parameters.

*Corresponding author: Qiong Wu, Department of Chemical Science and Technology, Kunming University, Kunming, Yunnan 65200, P. R. China, E-mail: wuqiongkm@163.com. https://orcid.org/0000-00017931-6750

Tianyu Li, Xindi Xu, Meifen Huang and Man Wang, Department of Chemical Science and Technology, Kunming University, Kunming, Yunnan 65200, P. R. China
Table 1: Data collection and handling.

\begin{tabular}{|c|c|}
\hline Crystal: & Black block \\
\hline Size: & $0.22 \times 0.20 \times 0.18 \mathrm{~mm}$ \\
\hline Wavelength: & Mo $K \alpha$ radiation $(0.71073 \AA)$ \\
\hline$\mu:$ & $1.27 \mathrm{~mm}^{-1}$ \\
\hline Diffractometer, scan mode: & Bruker APEX-II, $\varphi$ and $\omega$ \\
\hline$\theta_{\max }$, completeness: & $26.4^{\circ},>99 \%$ \\
\hline$N(h k l)_{\text {measured }}, N(h k l)_{\text {unique }}, R_{\text {int }}:$ & $22,308,2793,0.032$ \\
\hline Criterion for $I_{\mathrm{obs}}, N\left(h k l_{\mathrm{gt}}:\right.$ & $I_{\text {obs }}>2 \sigma\left(I_{\text {obs }}\right), 2500$ \\
\hline$N(\text { param })_{\text {refined }}:$ & 182 \\
\hline Programs: & $\begin{array}{l}\text { Bruker [1], Olex2 [2], } \\
\text { SHELX }[3,4]\end{array}$ \\
\hline
\end{tabular}

\section{Source of material}

All chemicals were commercially available and used without further purification. The title compound was synthesized with the following procedure: $0.0081 \mathrm{~mL} N^{\prime}, N$ bis(3-aminopropyl)methylamine $(0.05 \mathrm{mmol})$ was stirred in $20 \mathrm{~mL}$ methanol at room temperature for $5 \mathrm{~min}$. Then, $0.012 \mathrm{~g} \mathrm{CoCl}_{2} \cdot 6 \mathrm{H}_{2} \mathrm{O}(0.05 \mathrm{mmol})$ was added to the above solution and the resulting brown mixture was further stirred for another $30 \mathrm{~min}$. Then $0.05 \mathrm{~g} \mathrm{Ba}\left(\mathrm{N}_{3}\right)_{2}(0.23 \mathrm{mmol})$ was added and stirred for $1 \mathrm{~h}$ and filtered. The blocked reddish brown crystals of the title compound were obtained after three days by slow evaporation.

\section{Experimental details}

The structure was solved with the Olex2 program [2] as an interface together with the SHELXT and SHELXL programs $[3,4]$. All $\mathrm{H}$ atoms were placed in geometrically idealized positions and refined using a riding model.

\section{Comment}

The study on azide ligand have attracted increasing interest in the field of coodination chemistry and crystal engineering, not only because the predictable structures, but also the potential applications, such as magnetism and nonlinear optics $[5,6]$. 
Table 2: Fractional atomic coordinates and isotropic or equivalent isotropic displacement parameters $\left(\AA^{2}\right)$.

\begin{tabular}{|c|c|c|c|c|}
\hline Atom & $x$ & $y$ & $z$ & $U_{\text {iso }}{ }^{*} / U_{\text {eq }}$ \\
\hline C1 & $0.6913(3)$ & $0.49484(19)$ & $0.40998(12)$ & $0.0374(4)$ \\
\hline $\mathrm{H} 1 \mathrm{~A}$ & 0.607661 & 0.536987 & 0.430168 & $0.045^{\star}$ \\
\hline H1B & 0.784119 & 0.552650 & 0.407652 & $0.045^{\star}$ \\
\hline $\mathrm{C} 2$ & $0.7900(3)$ & $0.3924(2)$ & $0.46808(12)$ & $0.0420(5)$ \\
\hline $\mathrm{H} 2 \mathrm{~A}$ & 0.883174 & 0.425726 & 0.519391 & $0.050^{\star}$ \\
\hline $\mathrm{H} 2 \mathrm{~B}$ & 0.856404 & 0.342421 & 0.442922 & $0.050^{\star}$ \\
\hline C3 & $0.6518(3)$ & $0.3141(2)$ & $0.48757(13)$ & $0.0404(5)$ \\
\hline $\mathrm{H} 3 \mathrm{~A}$ & 0.573095 & 0.367065 & 0.504479 & $0.049^{\star}$ \\
\hline H3B & 0.723541 & 0.262885 & 0.534805 & $0.049^{\star}$ \\
\hline C5 & 0.3977 (3) & $0.1765(2)$ & $0.45522(13)$ & $0.0416(5)$ \\
\hline $\mathrm{H} 5 \mathrm{~A}$ & 0.341888 & 0.241306 & 0.475415 & $0.050^{*}$ \\
\hline H5B & 0.476465 & 0.128493 & 0.502983 & $0.050^{\star}$ \\
\hline C6 & $0.2380(3)$ & $0.0955(2)$ & 0.39753 (13) & $0.0427(5)$ \\
\hline $\mathrm{H} 6 \mathrm{~A}$ & 0.288220 & 0.039809 & 589 & $0.051^{*}$ \\
\hline H6B & 0.187761 & 0.047547 & 0.430368 & $0.051^{*}$ \\
\hline $\mathrm{C} 7$ & 0.0808 & $0.16932(17)$ & $0.33484(13)$ & 0.0359 (4) \\
\hline H7A & -0.027153 & 0.117070 & 0.305801 & $0.043^{\star}$ \\
\hline H7B & 0.041236 & 0.231953 & 0.363356 & $0.043^{*}$ \\
\hline $\mathrm{C} 8$ & $0.6419(3)$ & $0.1354(2)$ & 0.40584 (14) & $0.0462(5)$ \\
\hline H8A & 0.562283 & 076696 & 0.367003 & $0.069^{\star}$ \\
\hline H8B & 0.712787 & 0.096344 & 0.458099 & $0.069^{*}$ \\
\hline $\mathrm{H} 8 \mathrm{C}$ & 0.727722 & 0.170015 & 0.384553 & $0.069^{\star}$ \\
\hline Co1 & $0.37356(3)$ & $0.32842(2)$ & $0.30814(2)$ & $0.02593(9)$ \\
\hline N1 & $0.2817(2)$ & $0.43493(15)$ & $0.37329(10)$ & $0.0322(3)$ \\
\hline N2 & $0.1175(2)$ & $0.46119(13)$ & $0.34301(9)$ & 0.0299 (3) \\
\hline N3 & $-0.0416(2)$ & $0.48680(16)$ & $0.31647(11)$ & $0.0373(4)$ \\
\hline N4 & $0.2337(2)$ & $0.42656(14)$ & $0.20873(10)$ & $0.0330(3)$ \\
\hline N5 & $0.1770(2)$ & $0.38121(15)$ & $0.14043(10)$ & 0.0351 (4) \\
\hline N6 & $0.1192(3)$ & $0.3438(2)$ & $0.07346(12)$ & $0.0558(5)$ \\
\hline N7 & $0.4575(2)$ & $0.21847(14)$ & $0.24158(10)$ & $0.0322(3)$ \\
\hline N8 & $0.5645(2)$ & $0.25360(14)$ & $0.21172(9)$ & $0.0328(3)$ \\
\hline N9 & $0.6675(3)$ & $0.2825(2)$ & $0.18226(12)$ & $0.0492(5)$ \\
\hline N10 & $0.5809(2)$ & $0.44538(14)$ & $0.32626(9)$ & $0.0302(3)$ \\
\hline $\mathrm{H} 10 \mathrm{~A}$ & 0.671699 & 0.405786 & 0.307209 & $0.036^{\star}$ \\
\hline $\mathrm{H} 10 \mathrm{~B}$ & 0.525849 & 0.515426 & 0.288849 & $0.036^{\star}$ \\
\hline N11 & $0.1436(2)$ & $0.22663(14)$ & $0.27400(9)$ & $0.0308(3)$ \\
\hline $\mathrm{H} 11 \mathrm{~A}$ & 0.036887 & 0.277338 & 0.236929 & $0.037^{\star}$ \\
\hline H11B & 0.159277 & 0.160138 & 0.239279 & $0.037^{*}$ \\
\hline N12 & $0.5229(2)$ & $0.23305(14)$ & $0.41799(9)$ & $0.0315(3)$ \\
\hline
\end{tabular}

The asymmetric unit of the title structure contains one Co(III) atom, one $N^{\prime}, N$-bis(3-aminopropyl)methylamine unit and three azide anions. The central Co(III) atom exhibits a distorted octahedral coordination geometry and is accomplished by three $\mathrm{N}$ atoms $(\mathrm{N} 10, \mathrm{~N} 11, \mathrm{~N} 12)$ from $N^{\prime}, N$ bis(3-aminopropyl) methylamine unit and three $\mathrm{N}$ atoms from azide anions (see the Figure). The bond lengths and angles are within the normal ranges and are comparable to related structures [7-9].

Author contribution: All the authors have accepted responsibility for the entire content of this submitted manuscript and approved submission.

Research funding: Fund for Less Developed Regions of the National Natural Science Foundation of China (No. 31760257); Joint Basic Research Program (partial) of Yunnan Local Undergraduate Universities (2017FH001002); The reserve Academic and Technical Leaders of Yunnan Province (2019HB098).

Conflict of interest statement: The authors declare no conflicts of interest regarding this article.

\section{References}

1. BRUKER. SAINT, APEX2 and SADABS; Bruker AXS Inc.: Madison, Wisconsin, USA, 2009.

2. Dolomanov O. V., Bourhis L. J., Gildea R. J., Howard J. A. K., Puschmann H. OLEX2: a complete structure solution, refinement and analysis program. J. Appl. Crystallogr. 2010, 42, 339-341.

3. Sheldrick G. A short history of SHELX. Acta Crystallogr. 2008, A64, 112-122.

4. Sheldrick G. Crystal structure refinement with SHELXL. Acta Crystallogr. 2015, C71, 3-8.

5. Li Y., Wu Q., Lecren L., Clérac R. Synthesis, structure and magnetism of new polynuclear transition metal aggregates assembled with Schiff-base ligand and anionic $\mathrm{N}$-donor ligands. J. Mol. Struct. 2008, 890, 339-345.

6. Wu Q., Pu Q., Wu Y., Shi H., He Y., Li J., Fan Q. POM-assisted synthesis of the first cyclohexanediamine-based Salen-type $\mathrm{Mn}$ III-dimer complexes. J. Coord. Chem. 2015, 68, 1010-1020.

7. Yoo H. S., Kim J. I., Yang N., Koh E. K., Park J. G., Hong C. S. Uncommon ferromagnetic interactions in a homometallic Co(II) chain bridged by a single end-to-end azide. Inorg. Chem. 2007, 46, 9054-9056.

8. Chun H., Bernal I. Triazidocobalt(III) complexes with tridentate amine ligands. Acta Crystallogr. 2000, 56, 1326-1329.

9. Salah S., Massoud S.S., Spell M., Haq Z.K., Mautner F.A. facCobalt(III)-azido complexes derived from substituted pyridylbased tridentate amines. Transition Met. Chem. 2014, 39, 585-591. 